\title{
Kinetics of testosterone recovery in clinically localized prostate cancer patients treated with radical prostatectomy and subsequent short-term adjuvant androgen deprivation therapy
}

\author{
Bo Dai ${ }^{1,2, *}$, Yuan-Yuan Qu ${ }^{1,2, *}$, Yun-Yi Kong ${ }^{2,3}$, Ding-Wei Ye ${ }^{1,2}$, Xu-Dong Yao ${ }^{1,2}$, Shi-Lin Zhang ${ }^{1,2}$, \\ Hai-Liang Zhang ${ }^{1,2}$ and Wei-Yi Yang ${ }^{1,2}$
}

Androgen deprivation therapy (ADT) is a standard treatment for metastatic, recurrent and locally advanced prostate cancer (PCa). The aim of this study is to investigate the timing and extent of testosterone recovery in clinically localized PCa patients treated with radical prostatectomy (RP) and subsequent short-term adjuvant ADT. A total of 95 localized PCa patients underwent RP and 9-month adjuvant ADT were included in this prospective study. Serum testosterone level was measured before adjuvant ADT, at ADT cessation, and at 1 , 3, 6, 9 and 12 months after cessation of ADT. A Cox proportional hazards model was used to assess variables associated with the time of testosterone normalization. The results showed that median patient age was 67 years and median testosterone level before adjuvant ADT was 361 (230-905) $\mathrm{ng} \mathrm{dl}^{-1}$. All patients finished 9-month adjuvant ADT and achieved castrate testosterone level. At 3 months after ADT cessation, testosterone recovered to supracastrate level in $97.9 \%$ patients and to normal level in $36.9 \%$ patients. The percentage of patients who recovered to normal testosterone level increased to $66.3 \%, 86.3 \%$ and $92.6 \%$ at 6,9 and 12 months, respectively. Cox regression model found that higher baseline testosterone level ( $\geqslant 300 \mathrm{ng} \mathrm{dl}^{-1}$ ) was the only variable associated with a shorter time to testosterone normalization (hazard ratio: $1.98 ; P=0.012$ ). In conclusion, in most patients, testosterone recovered to supracastrate level at 3 months and to normal level at 12 months after 9-month adjuvant ADT cessation. Patients with higher baseline testosterone level need shorter time of testosterone normalization.

Asian Journal of Andrology (2013) 15, 466-470; doi:10.1038/aja.2012.169; published online 27 May 2013

Keywords: androgen deprivation therapy (ADT); luteinizing hormone-releasing hormone; prostatic neoplasms; recovery of function; testosterone

\section{INTRODUCTION}

Androgen deprivation therapy (ADT) is a standard treatment for metastatic, recurrent and locally advanced prostate cancer (PCa). Luteinizing hormone-releasing hormone (LHRH) agonists are widely used for ADT because it allows for testosterone recovery after its cessation. After the administration of LHRH agonists, castrate levels of testosterone are achieved in 2-4 weeks. ${ }^{1,2}$ However, the factors which influence the time course and extent of testosterone recovery after the cessation of LHRH agonists are poorly understood. Most previous studies investigating this issue are retrospectively designed and have some limitations, including (i) devoid of measuring baseline testosterone level before ADT; (ii) inconsistency of ADT duration in the same study; and (iii) lack of considering the effect of radiation therapy on testosterone recovery. ${ }^{3-10}$ Therefore, the results from previous studies are controversial and confusing.

The role of adjuvant ADT is still controversial for patients treated with radical prostatectomy (RP). In a prospective, randomized trial, a survival benefit with adjuvant ADT in patients with lymph node positive disease was shown. ${ }^{11}$ In addition, the ADT alone control arm of SWOG (Southwest Oncology Group) study S9921 demonstrated excellent 5-year progression-free (92.5\%) and overall survival rates (95.9\%) for men with high-risk PCa treated with RP and 2-year period (long-term) adjuvant ADT. ${ }^{12}$ These excellent results suggest that there might be a role for adjuvant ADT in men with pT3 disease and/or positive surgical margins. Moreover, the increasing evidence of ADT related toxicities led us to think about whether the short-term adjuvant $\mathrm{ADT}$ has the same effect as the long-term adjuvant ADT. Therefore, we conducted a prospective study in high-risk clinically localized PCa patients to investigate the efficacy and safety of a combined approach of RP plus subsequent short-term ( 9 months) adjuvant ADT. Although the survival data are not yet mature, we summarize the currently available data to investigate the timing and extent of testosterone recovery after short-term ADT cessation in this study. 


\section{MATERIALS AND METHODS}

\section{Patients}

The current study was designed to investigate the efficacy and safety of a combined approach of RP plus subsequent short-term (9 months) adjuvant ADT for high-risk clinically localized PCa patients. From January 2006 to July 2010, 95 Chinese clinically localized high-risk PCa patients were included in this study at our institution. All patients underwent open retropubic RP and had at least one of the following poor prognostic factors: pathological Gleason score $\geqslant 8$, pathological stage $\geqslant \mathrm{pT} 3 \mathrm{a}$ or positive surgical margins. Patients with positive pelvic lymph nodes were excluded from this study. No patient received any kind of neoadjuvant hormonal therapy before RP. Histological slides of all prostatectomy specimens were assessed by two dedicated genitourinary pathologists. Prostate-specific antigen (PSA) levels were measured 1 day before RP and at 6 weeks after RP in all patients. Adjuvant ADT started at 6 weeks post-RP after the postoperative PSA level was measured. All patients had normal serum testosterone $\left(\geqslant 230 \mathrm{ng} \mathrm{dl}^{-1}\right)$ before the start of adjuvant ADT. Short-term adjuvant ADT lasted for 9 months and consisted of goserelin acetate $3.6 \mathrm{mg}$ subcutaneously or triptorelin acetate $3.75 \mathrm{mg}$ intramuscularly every month, and bicalutamide $50 \mathrm{mg}$ orally once a day. Patients were excluded if they received any kind of radiation therapy before or after RP. This study was carried out in accordance with the ethical standards of the Helsinki Declaration II and approved by the Institutional Review Board of our institution. A written informed consent was obtained from each patient before any study-specific investigation was done.

\section{Testosterone assay}

Serum testosterone level in all samples was detected in the same laboratory using Access Testosterone (Beckman Coulter, Fullerton, CA, USA) based on electrochemiluminescence immunoassay technique. The assay was used according to the manufacturer's recommendations. A serum testosterone level lower than $50 \mathrm{ng} \mathrm{dl}^{-1}$ was considered as castrate level, and equal or greater than $230 \mathrm{ng} \mathrm{dl}^{-1}$ was considered as normal. Testosterone level was measured before the start of adjuvant $\mathrm{ADT}$ to ensure all patients included in this study had normal testosterone at baseline. Testosterone and PSA were measured at the time of adjuvant ADT cessation, and subsequently at 1, 3, 6, 9 and 12 months after the cessation of ADT.

\section{Statistical analysis}

Descriptive statistics were used to characterize the study cohort. A Cox proportional hazards model was used to assess whether age ( $<65$ years $v s . \geqslant 65$ years $)$, preoperative PSA level $\left(<20 \mathrm{ng} \mathrm{ml}^{-1} v s . \geqslant 20 \mathrm{ng} \mathrm{ml}^{-1}\right)$, baseline testosterone level $\left(<300 \mathrm{ng} \mathrm{dl}^{-1}\right.$ vs. $\left.\geqslant 300 \mathrm{ng} \mathrm{dl}^{-1}\right)$, pathological Gleason score $(<8$ vs. $8-10)$ and pathological stage (T2 vs. T3) were associated with the time of testosterone normalization. The hazard ratio for the time of testosterone normalization with associated $95 \%$ confidence interval (CI) and two-sided $P$ values were calculated for each variable. SPSS 16.0 (SPSS, Chicago, IL, USA) was used for the statistical analysis and $P<0.05$ was considered statistically significant.

\section{RESULTS}

Patients' characteristics and baseline data are listed in Table 1. The median patient age was 67 years old and the median patient body mass index was $24.2 \mathrm{~kg} \mathrm{~m}^{-2}$. In prostatectomy specimens, Gleason score $\geqslant 8$ were observed in $52.6 \%$ patients and pathologic stage $\geqslant$ pT3a was found in $80.0 \%$ patients. The median PSA level before RP was $12.5 \mathrm{ng}$ $\mathrm{ml}^{-1}$. Before adjuvant ADT at 6 weeks after RP, the median PSA level decreased to $0.049 \mathrm{ng} \mathrm{ml}^{-1}$ and $89.5 \%$ (85/95) patients achieved a PSA
Table 1 Clinicopathological characteristics of 95 patients

\begin{tabular}{|c|c|c|}
\hline Characteristics & Mean (median) & Range \\
\hline Age before start of ADT (year) & $66.7(67.0)$ & 51.0-78.0 \\
\hline Weight (kg) & $69.3(70.0)$ & $51.0-103.0$ \\
\hline Height (m) & $169.8(170.0)$ & 150.0-191.0 \\
\hline $\mathrm{BMI}\left(\mathrm{kg} \mathrm{m}^{-2}\right)$ & $24.1(24.2)$ & $19.9-29.8$ \\
\hline PSA before RP (ng ml ${ }^{-1}$ ) & $26.2(12.5)$ & $3.1-156.3$ \\
\hline PSA post-RP (ng ml $\left.{ }^{-1}\right)$ & $0.068(0.049)$ & $0.003-0.871$ \\
\hline $\begin{array}{l}\text { Testosterone before start of ADT } \\
\left(\mathrm{ng} \mathrm{dl}^{-1}\right)\end{array}$ & $429(361)$ & 230-905 \\
\hline \multicolumn{3}{|l|}{ Duration of ADT, $n(\%)$} \\
\hline 9 months & $95(100)$ & \\
\hline \multicolumn{3}{|l|}{ Pathological Gleason score, $n(\%)$} \\
\hline$\leqslant 6$ & $9(9.5)$ & \\
\hline 7 & 36 (37.9) & \\
\hline$\geqslant 8$ & $50(52.6)$ & \\
\hline \multicolumn{3}{|l|}{ Pathological tumor stage, $n(\%)$} \\
\hline T2a-T2b & 0 & \\
\hline $\mathrm{T} 2 \mathrm{c}$ & $19(20.0)$ & \\
\hline ТЗа & $45(47.4)$ & \\
\hline T3b & $31(32.6)$ & \\
\hline \multicolumn{3}{|l|}{ Positive surgical margins, $n(\%)$} \\
\hline No & $59(62.1)$ & \\
\hline Yes & 36 (37.9) & \\
\hline
\end{tabular}

Abbreviations: ADT, androgen deprivation therapy; BMI, body mass index; PSA, prostate-specific antigen; RP, radical prostatectomy.

level $<0.1 \mathrm{ng} \mathrm{ml}^{-1}$. The serum testosterone level before start of adjuvant ADT ranged from 230 to $905 \mathrm{ng} \mathrm{dl}^{-1}$. All patients finished 9month adjuvant $\mathrm{ADT}$ and achieved castrate testosterone level at the time of ADT cessation. After the cessation of adjuvant ADT, one patient developed biochemical recurrence (PSA level greater than $\left.0.2 \mathrm{ng} \mathrm{ml}^{-1}\right) 9$ months later and the remaining 94 patients were in good health without any relapse of prostate cancer at the end of this study.

As shown in Figure 1, the median serum testosterone level at 0, 1, 3, 6, 9 and 12 months after the cessation of ADT was 19.5, 47.5, 257.0, $316.5,355.0$ and $384.5 \mathrm{ng} \mathrm{dl}^{-1}$, respectively. Figure 2 depicts the cumulative incidence for testosterone recovery to supracastrate level and normal level after cessation of adjuvant ADT. At 1 month after cessation, testosterone recovered to supracastrate level in 43.2\% (41/ 95) patients and no patient had his testosterone recovered to normal range. At 3 months after cessation, testosterone recovered to supracastrate level in $97.9 \%$ (93/95) patients and to normal range in $36.8 \%$ (35/95) patients. All patients recovered to supracastrate testosterone level at 6 months after ADT cessation and maintained to the end of this study. The cumulative incidence of patients whose testosterone level recovered to normal range increased to $66.3 \%$ (63/95), 86.3\% (82/95) and $92.6 \%(88 / 95)$ at 6,9 and 12 months, respectively.

On multivariate analysis (Table 2) with a Cox regression model including age, PSA before RP, baseline testosterone level, pathological Gleason score and pathological stage, higher baseline testosterone level $\left(\geqslant 300 \mathrm{ng} \mathrm{dl}^{-1}\right)$ was the only variable associated with a shorter time to testosterone normalization (HR: 1.98; 95\% CI: $1.17-3.37 ; P=0.012$ ). Figures 3 shows cumulative incidence curves for recovery to normal testosterone level according to baseline testosterone level $(<300 \mathrm{ng}$ $\mathrm{dl}^{-1}$ vs. $\geqslant 300 \mathrm{ng} \mathrm{dl}^{-1}$ ).

\section{DISCUSSION}

The present study is the first prospective trial that investigated the factors associated with testosterone recovery after ADT cessation in 


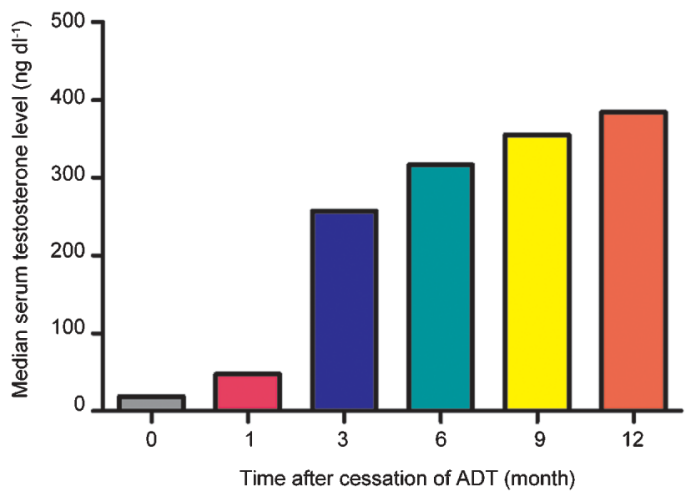

Figure 1 The median serum testosterone level after cessation of adjuvant ADT. $\mathrm{ADT}$, androgen deprivation therapy.

clinically localized PCa patients treated by RP and subsequent shortterm (lasted 9 months) adjuvant ADT. In this study, we have found that 6 months after the cessation of short-term adjuvant ADT, testosterone recovered to supracastrate level and normal range in $100 \%$ and $66.3 \%$ patients, respectively. As time went by, $92.6 \%$ patients recovered to normal level at 12 months after the cessation of adjuvant ADT. In addition, multivariate analysis showed that higher baseline testosterone level before ADT is correlated with a shorter time of testosterone normalization after ADT cessation. However, we did not find a correlation of time of testosterone normalization with patient age, PSA before RP, pathological cancer grade and stage.

Over the past decade, increasing evidence suggested that ADT, which leads to castrate testosterone level, is associated with numerous side effects, such as osteoporosis, obesity, sarcopenia, lipid alterations, insulin resistance and increased risk for diabetes and cardiovascular morbidity. ${ }^{13}$ Moreover, serum PSA, which is widely used for early detection and monitoring the treatment response of $\mathrm{PCa}$, is strongly androgen-dependent. Therefore, studies investigating the timing and extent of testosterone recovery after ADT cessation in PCa patients should focus on two crucial issues: first, time of testosterone recovery to supracastrate level, and second, time of testosterone recovery to normal level. Once testosterone recovered to supracastrate level, it

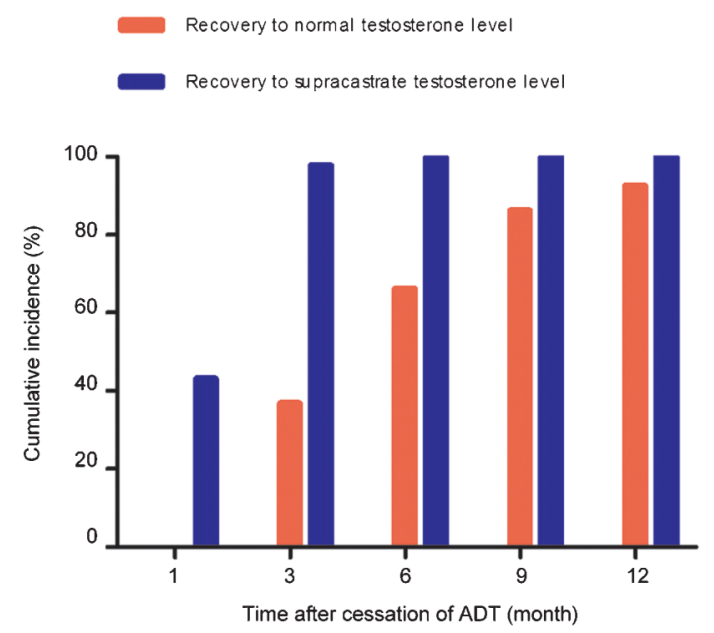

Figure 2 The cumulative incidence of patients recovering to above castrate and normal testosterone level after cessation of adjuvant ADT. ADT, androgen deprivation therapy. would influence serum PSA level and the interpretation of PSA relapse rates in clinical studies involving LHRH agonists. After testosterone recovered to normal level, the side effects of ADT would disappear completely. ${ }^{13,14}$

Time of testosterone recovery to supracastrate level after ADT has been investigated in previous studies. In a randomized clinical trial using intermittent hormonal therapy for PCa patients with increasing PSA after definitive local treatment, Gulley et al. ${ }^{6}$ found that after 6 months of ADT, more than $90 \%$ patients achieved supracastrate serum testosterone level by 18 weeks. In another study Murthy and colleagues ${ }^{8}$ found that after short-course (lasted 54-194 days) neoadjuvant ADT and radical radiotherapy in localized $\mathrm{PCa}$, all patients recovered to supracastrate testosterone level by 12 weeks after the last LHRH agonists injection. Results from the present study showed that $97.9 \%$ and $100 \%$ patients could recover to supracastrate testosterone level at 3 and 6 months after cessation of 9 -month ADT. Therefore, it is not difficult for PCa patients to recover to supracastrate testosterone level after short-term ADT therapy, because more than $90 \%$ patients could achieve this goal within 3 months. However, when it comes to long-term $\mathrm{ADT}$, things become complicated. In a study which included 32 patients who received ADT for a minimum of 2 years (24-87 months) as an adjuvant therapy for RP or external beam radiation therapy (EBRT), Kaku et al. ${ }^{7}$ revealed that it took 30 months for more than $90 \%$ patients to recover to supracastrate testosterone level. Yoon et al. ${ }^{4}$ also reported that after 2 years of adjuvant ADT for PCa patients underwent RP, it took 36 months for $93.2 \%$ patients to recover to supracastrate testosterone level. These data suggested that after long-term ADT, it takes a few years for most patients to recover to supracastrate testosterone level.

Previous studies did not solve the issue concerning time of testosterone recovery to normal level after ADT successfully, because (i) the definition of normal testosterone level varied among these studies; and (ii) few studies measured baseline testosterone level before ADT but none of them excluded patients without normal baseline testosterone. ${ }^{3-9}$ It has been reported that if a patient had baseline testosterone level below the normal range, it is difficult for him to recover to normal range after the cessation of $\mathrm{ADT}^{4}$ The first strength of the present study is that we defined a serum testosterone level $\geqslant 230 \mathrm{ng} \mathrm{dl}^{-1}$ as normal and excluded patients whose baseline testosterone lower than normal level. The reason for defining testosterone $\geqslant 230 \mathrm{ng} \mathrm{dl}^{-1}$ as normal is that International Society of Andrology, European Association of Urology, European Academy of Andrology and American Society of Andrology recommendations suggest that patients with serum testosterone levels below $230 \mathrm{ng} \mathrm{dl}^{-1}(8 \mathrm{nmol}$ $1^{-1}$ ) will usually benefit from testosterone replacement treatment. ${ }^{15,16}$ Previously, Gulley et al. ${ }^{6}$ defined testosterone $\geqslant 212 \mathrm{ng} \mathrm{dl}^{-1}$ as normal and found that $80 \%$ patients achieved this level at 6 months after short-term (6 months) ADT. ${ }^{6}$ In another study Murthy et al. ${ }^{8}$ defined testosterone $\geqslant 6 \mathrm{nmol} \mathrm{l}^{-1}\left(173 \mathrm{ng} \mathrm{dl}^{-1}\right)$ as normal and reported that after short-term (54-194 days) neoadjuvant ADT and EBRT, 89\% patients recovered to normal range at 6 months. However, in this study, only $66.3 \%$ patients recovered to normal testosterone level at 6 months after the cessation of short-term (9 months) adjuvant ADT. This may be due to the comparatively longer period of ADT and different definition of normal testosterone level in this study. The second strength of this study is that we excluded patients who received any kind of radiation therapy before or after RP. Previous studies revealed that EBRT for PCa leads to impaired spermatogenesis and depressed testosterone production, because the routine EBRT dose for treating $\mathrm{PCa}$ is $78 \mathrm{~Gy}$, which results in approximately 2 Gy radiation 
Table 2 Multivariate Cox regression model for time to normalization of testosterone

\begin{tabular}{lcc}
\hline Variables & Hazard ratio $(95 \% \mathrm{Cl})$ & $\mathrm{P}$ \\
\hline Age $(<65$ years vs. $\geqslant 65$ years $)$ & $0.62(0.35-1.09)$ & 0.096 \\
PSA $\left(<20 \mathrm{ng} \mathrm{ml}^{-1}\right.$ vs. $\left.\geqslant 20 \mathrm{ng} \mathrm{ml}^{-1}\right)$ & $0.74(0.42-1.30)$ & 0.289 \\
Baseline testosterone $\left(<300 \mathrm{ng} \mathrm{dl}^{-1}\right.$ vs. $\left.\geqslant 300 \mathrm{ng} \mathrm{dl}^{-1}\right)$ & $1.98(1.17-3.37)$ & 0.012 \\
Pathological Gleason score $(<8$ vs. 8-10) & $0.81(0.47-1.40)$ & 0.456 \\
Pathological stage (T2 vs. T3) & $0.91(0.51-1.63)$ & 0.742 \\
\hline
\end{tabular}

Abbreviation: PSA, prostate-specific antigen.

scattered to unshielded testes. ${ }^{17,18}$ Recently, Zagars et al. ${ }^{19}$ demonstrated a statistically significant decrease in testosterone at 3 months after EBRT, and Daniell et al. ${ }^{20}$ reported lowered testosterone levels at 3-8 years after EBRT compared with patients treated with RP. Most previous studies which investigated testosterone recovery included patients treated by ADT combined with EBRT and did not set a control group receiving EBRT alone. ${ }^{3-9}$ Therefore, they could not separate the effects of ADT on the extent and timing of testosterone recovery from that of EBRT plus ADT. ${ }^{3-9}$ Benefit from the design of this study, testosterone recovery in our patients bears only the effect of 9-month adjuvant ADT.

Factors including age before ADT, duration of ADT, baseline testosterone level, baseline dihydroxytestosterone level, race and Gleason score at diagnosis have been reported be correlated with the timing and extent of testosterone recovery after ADT in previous studies. $^{3-7,9,21,22}$ Therefore, it is not surprising that a correlation between patient baseline testosterone level and timing of testosterone normalization was found in this study. We failed to demonstrate a correlation between age and timing of testosterone normalization in our patients. It may be due to that all patients included in this study had normal baseline testosterone level. Previous studies found that younger age was associated with a faster recovery of testosterone and gave the plausible explanation which was the decreased ability of older men to produce and secrete testosterone. ${ }^{4,5}$ If patients who had pre-existing dysfunctional Leydig cells and subnormal baseline testosterone level were excluded, the association between age and testosterone recovery may disappear in these studies.

Our study also has several limitations. One limitation was that the blood samples for testosterone test were not drawn at the same time each day. Therefore, we could not avoid the influence of diurnal variation effect on serum testosterone level. The second limitation was that we did not evaluate the side effects of ADT in our patients and could not determine whether the side effects were disappeared after testosterone recovered to normal level.

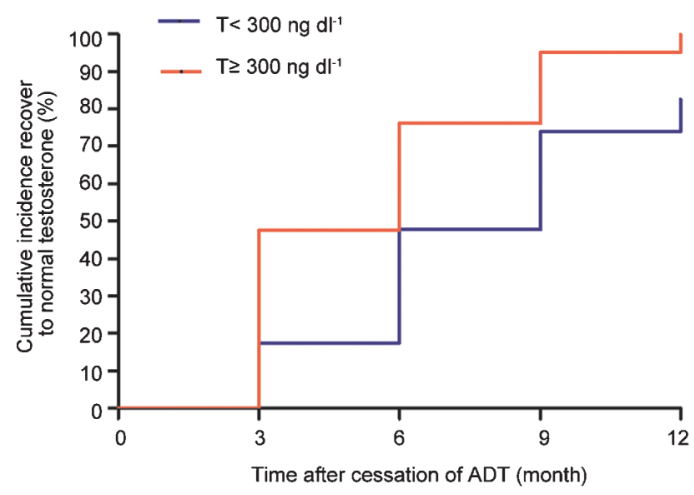

Figure 3 The cumulative incidence curves of recovery to normal testosterone level by baseline testosterone level ( $<300 \mathrm{ng} \mathrm{dl}^{-1}$ vs. $\geqslant 300 \mathrm{ng} \mathrm{dl}^{-1}$ ).

\section{CONCLUSIONS}

This prospective study shows that in most clinically localized PCa patients treated by RP and subsequent short-term ( 9 months) adjuvant $\mathrm{ADT}$, serum testosterone can recovery to supracastrate level at 3 months after ADT cessation and to normal level at 12 months after ADT cessation. Moreover, patients with higher baseline testosterone $\left(\geqslant 300 \mathrm{ng} \mathrm{dl}^{-1}\right)$ level need shorter time of testosterone normalization after adjuvant ADT cessation.

\section{AUTHOR CONTRIBUTIONS}

$\mathrm{BD}$ and YYQ contributed to conception, design, acquisition, analysis and interpretation of data, drafting the manuscript, critical revision for important intellectual content and final approval of the version to be published. YYK contributed to analysis and interpretation of data. DWY contributed to conception, interpretation of data and final approval of the version to be published. XDY, SLZ, HLZ and WYY contributed to acquisition of data.

\section{COMPETING FINANCIAL INTERESTS}

The authors declare that they have no financial or commercial interests related to this study.

\section{ACKNOWLEDGMENTS}

This study was supported by Grants for International Cooperation and Exchange of Science and Technology Commission of Shanghai Municipality (No. 12410709300) and Grant from Guide Project of Science and Technology Commission of Shanghai Municipality (No. 124119a7300).

1 Anderson J, Abrahamsson PA, Crawford D, Miller K, Tombal B. Management of advanced prostate cancer: can we improve on androgen deprivation therapy? BJU Int 2008; 101: 1497-501.

2 Perachino M, Cavalli V, Bravi F. Testosterone levels in patients with metastatic prostate cancer treated with luteinizing hormone-releasing hormone therapy: prognostic significance? BJU Int 2010; 105: 648-51.

3 Pickles T, Agranovich A, Berthelet E, Duncan GG, Keyes M et al. Testosterone recovery following prolonged adjuvant androgen ablation for prostate carcinoma. Cancer 2002; 94: 362-7.

4 Yoon FH, Gardner SL, Danjoux C, Morton G, Cheung P et al. Testosterone recovery after prolonged androgen suppression in patients with prostate cancer. J Urol 2008; 80: 1438-43; discussion 1443-4.

5 Bong GW, Clarke HS Jr, Hancock WC, Keane TE. Serum testosterone recovery after cessation of long-term luteinizing hormone-releasing hormone agonist in patients with prostate cancer. Urology 2008; 71: 1177-80.

6 Gulley JL, Figg WD, Steinberg SM, Carter J, Sartor 0 et al. A prospective analysis of the time to normalization of serum androgens following 6 months of androgen deprivation therapy in patients on a randomized phase III clinical trial using limited hormonal therapy. J Urol 2005; 173: 1567-71.

7 Kaku H, Saika T, Tsushima T, Ebara S, Senoh T et al. Time course of serum testosterone and luteinizing hormone levels after cessation of long-term luteinizing hormone-releasing hormone agonist treatment in patients with prostate cancer. Prostate 2006; 66: 439-44.

8 Murthy V, Norman AR, Shahidi M, Parker CC, Horwich A et al. Recovery of serum testosterone after neoadjuvant androgen deprivation therapy and radical radiotherapy in localized prostate cancer. BJU Int 2006; 97: 476-9.

9 Wilke DR, Parker C, Andonowski A, Tsuji D, Catton C et al. Testosterone and erectile function recovery after radiotherapy and long-term androgen deprivation with luteinizing hormone-releasing hormone agonists. BJU Int 2006; 97: 963-8. 
10 Spry NA, Galvao DA, Davies R, La Bianca S, Joseph D et al. Long-term effects of intermittent androgen suppression on testosterone recovery and bone mineral density: results of a 33-month observational study. BJU Int 2009; 104: 806-12.

11 Messing EM, Manola J, Yao J, Kiernan M, Crawford D et al. Immediate versus deferred androgen deprivation treatment in patients with node-positive prostate cancer after radical prostatectomy and pelvic lymphadenectomy. Lancet Oncol 2006; 7: 472-9.

12 Dorff TB, Flaig TW, Tangen CM, Hussain MH, Swanson GP et al. Adjuvant androgen deprivation for high-risk prostate cancer after radical prostatectomy: SWOG S9921 study. J Clin Oncol 2011; 29: 2040-5.

13 Isbarn H, Boccon-Gibod L, Carroll PR, Montorsi F, Schulman C et al. Androgen deprivation therapy for the treatment of prostate cancer: consider both benefits and risks. Eur Urol 2009; 55: 62-75.

14 Abrahamsson PA. Potential benefits of intermittent androgen suppression therapy in the treatment of prostate cancer: a systematic review of the literature. Eur Urol 2010; 57: 49-59.

15 Wang C, Nieschlag E, Swerdloff R, Behre HM, Hellstrom WJ et al. Investigation, treatment, and monitoring of late-onset hypogonadism in males: ISA, ISSAM, EAU, EAA, and ASA recommendations. Eur Urol 2009; 55: 121-30.
16 Dandona P, Rosenberg MT. A practical guide to male hypogonadism in the primary care setting. Int J Clin Pract 2010; 64: 682-96.

17 Grigsby PW, Perez CA. The effects of external beam radiotherapy on endocrine function in patients with carcinoma of the prostate. J Urol 1986; 135: 726-7.

18 Budgell GJ, Cowan RA, Hounsell AR. Prediction of scattered dose to the testes in abdominopelvic radiotherapy. Clin Oncol (R Coll Radiol) 2001; 13: 120-5.

19 Zagars GK, Pollack A. Serum testosterone levels after external beam radiation for clinically localized prostate cancer. Int J Radiat Oncol Biol Phys 1997; 39: 85-9.

20 Daniell HW, Clark JC, Pereira SE, Niazi ZA, Ferguson DW et al. Hypogonadism following prostate-bed radiation therapy for prostate carcinoma. Cancer 2001; 91: 1889-95.

21 Gulley JL, Aragon-Ching JB, Steinberg SM, Hussain MH, Sartor 0 et al. Kinetics of serum androgen normalization and factors associated with testosterone reserve after limited androgen deprivation therapy for nonmetastatic prostate cancer. J Urol 2008; 180: 1432-7; discussion 1437.

22 Martin NE, Chen MH, Nguyen PL, Beard CJ, Loffredo MJ et al. Biopsy Gleason score and the duration of testosterone suppression among men treated with external beam radiation and 6 months of combined androgen blockade. BJU Int 2012: 110: 1252-6. 